\title{
State-of-the-art on Virtualization and Software Defined Networking for Efficient Resource Allocation on Multi-tenant 5G Networks
}

\author{
Christos Tsirakis ${ }^{1 *}$, Panagiotis Matzoros ${ }^{1}$, and George Agapiou ${ }^{2}$ \\ ${ }^{1}$ OTE Academy S.A., Maroussi-Athens, Greece \\ ${ }^{2}$ OTE S.A., Maroussi-Athens, Greece
}

\begin{abstract}
Global data traffic explosion is expected to set stringent requirements for next generation networks in the next decades. Besides, very low latencies will have to be guaranteed for enabling new delay critical services. However, current Software Defined Networking (SDN) solutions have limitations in terms of separating both data and control planes among tenants/operators, and the capability to adapt to new or changing requirements. Moreover, some virtualization schemes do not ensure isolation of resources and do not guarantee bandwidth across the entities. While some others fail to provide flexibility to the slices to customize the resource allocation across the users. Therefore, novel SDN and virtualization techniques should be implemented to realize the upcoming $5 \mathrm{G}$ network that will facilitate at least efficient resource allocation and multi-tenancy among the plethora of different requirements.
\end{abstract}

\section{Introduction}

Currently, mobile networks are a key element of society, enabling communication, access and information sharing. In future, the number of smart devices connected to the Internet is projected to expand to somewhere between 20 and 46 billion by 2020 , and in turn the mobile data traffic, that these smart devices generate, will determine a 1000 -fold capacity increase by 2020 [1]. In addition, except from the constantly increasing traffic demand from the end users, one of the major concerns of wireless networks comes from the spectrum scarcity. Studies show that spectrum resources owned by a single operator are often underutilized; as in [2], where macro-cell utilization seems to be typically around $20-40 \%$. Thus, current wireless and mobile networks should evolve to become more intelligent, efficient, secure, and extremely scalable to deal with a torrent of data communications without deteriorating the quality and reliability of the provided services and effectively reducing capital and operational costs if possible.

On the one hand, this challenge has led to the consideration of new access technologies or the need to improve the efficiency of the existing ones. Paradigms such as Heterogeneous Networks (HetNets) (e.g., femtocells and small cells), the combination of different Radio Access Technologies (RATs) and the use of the cognitive radio concept have appeared as candidate alternatives to increase the efficiency of wireless networks. On the other hand, these paradigms will potentially increase the costs of network operators (CapEx and OpEx) by requiring the deployment of more infrastructure, and consequently making network management more complex [3].

Not long ago, the fifth generation $(5 \mathrm{G})$ cellular network was defined and discussed globally. The 5GPPP group released its plan and perspectives for future mobile networks until 2020. It considers virtualization and SDN to be two major trends in the evolution of the mobile network and serve as key enablers for future $5 \mathrm{G}$ cellular networks $[4,5]$. There is a close relationship between virtualization and SDN. They are mutually beneficial, highly complementary to each other, and share the same feature of promoting innovation, creativity, openness and competitiveness [6].

However, it is emphasized that the virtualization and deployment of network functions do not necessarily rely on SDN technologies, and vice versa [7]. While both network virtualization and SDN manage networks, they rely on different methods. While SDN separates the control and data/forwarding planes to offer a centralized view of the network, virtualization is a complementary approach to SDN for network management primarily and focuses on optimizing the network services themselves.

Indeed, due to the strong coupling between control and data planes (and its physical embedding in the network elements) in conventional networks, the development and deployment of new networking features would imply a modification of the control plane of all network devices through the installation of new firmware and, in some cases, hardware upgrades. This approach would imply very long periods of deployment and unaffordable costs, therefore the new networking features are commonly introduced via expensive, specialized, and hard-to-configure equipment (also

ctsirakis@oteacademy.gr 
known as middleboxes). Also, the centralization of the control logic in a controller with global knowledge of the network state simplifies the development of more sophisticated networking functions (e.g., routing algorithms), services, and applications.

In that sense, the network virtualization is a promising solution that will allow for realizing the vision of $5 \mathrm{G}$ and includes many advantages [9]. First of all, it has the advantage of separating the physical infrastructure from its services. More specifically, physical mobile network infrastructure resources, such as radio access networks (RANs), core networks (CNs), and physical radio resources (licensed spectrum), can be abstracted and sliced into virtual cellular network resources, and shared by multiple tenants/operators through isolating each other. As a result, network infrastructure can be decoupled from the services it provides, and differentiated/customized services can be provided to customers, enhancing the Quality of Service (QoS) management. Also, virtualization enables the hostage of multiple virtual base stations on a physical one, so there is no need to deploy new infrastructure and avoid payments for constructing new base stations and their maintenance. Furthermore, efficient resource utilization is achieved by keeping the scarce wireless channels occupied as much as possible when allowing usage of unused resources by one entity to other entities and assigning wireless resources intelligently based on the actual need [10].

However, wireless network virtualization (WNV), in comparison with wired network virtualization, introduces a number of challenges that do not exist in the wired domain: signal propagation, interference, user mobility, radio access technology. Wireless links are less reliable, suffer from interference and have a fluctuating capacity depending on the channel quality. All these challenges make the problem more complicated and will have to be taken into account when developing a proper solution to meet the expected requirements [11].

In this context, a key business model for reducing future deployment and operational costs is network sharing. There are different approaches to network sharing:

1) Spectrum sharing: It refers to the licensed spectrum owned by operators that can be utilized by multiple contracted operators based on agreements. The total available radio spectrum is considered as a whole resource and is virtualized as the abstracted access medium.

2) Infrastructure sharing: Only infrastructures are shared in this case. Infrastructure sharing can be classified into two categories: (a) passive sharing and (b) active sharing. Passive sharing refers to the reuse of components such as physical sites, tower masts, cabling, cabinets, power supply, and so on. Active RAN sharing involves sharing base stations among multiple mobile virtual network operators (MVNOs) with either separate spectrum resources for each entity or shared spectrum resources through spectrum pooling.

3) Full network sharing: It is the combination of spectrum sharing and infrastructure sharing, which means both radio resource and network infrastructure can be shared among multiple mobile network operators (MNOs) based on agreements.

Thus, a survey of resource sharing deployments is a key building block for virtualizing future mobile networks in order to address the explosive capacity demand of mobile traffic, reduce infrastructure investments, and enhancing the overall resource utilization by utilizing the wireless resources more efficiently. In this work, state-of-the-art virtualization and SDN solutions, that attempt to address the aforementioned challenges and meet the requirements of the upcoming $5 \mathrm{G}$ technology, are presented.

\section{Virtualization and Resource Allocation}

This work focuses more on RAN virtualization and ways to efficiently share the available spectrum among multiple tenants. In fact, when implementing slicing on a wireless network, the main issue is how to assign resources to the different slices [11]. This is known as the resource allocation problem. In this section, we present current proposals for resource allocation and multi-tenancy support in wireless networks, and explain the characteristics of these solutions.

As already mentioned, the WNV concept can be applied at different layers and degrees, from only virtualizing the core network to virtualizing the radio spectrum and physical layer of base stations (BSs).

One option for the implementation of spectrum virtualization could be to share the RF front end and antenna of the BS, like in [12], where the flexible slicing of a radio into multiple slices, each operating on different spectrum fragments, is enabled. Modifying the scheduling software in use is another option. In fact, the vast majority of approaches modify the frame scheduler to assign Physical Resource Blocks (PRBs) to the slices $[13,14,15$, and 16]. The PRB structure is described in [13]. As a result, BS virtualization can allow each tenant to have its own customized schedulers over its slice.

The BS is the entity responsible for accessing the radio channel and scheduling the air interface resources between the users. In order to effectively allocate resources, these should be virtualized first. Therefore, the BS has to be virtualized to virtualize in turn the air interface. Virtualizing the BS is similar to node virtualization. The physical resources of the node (e.g., CPU, memory, I/O devices) are shared between multiple virtual instances. A hypervisor, which is a well-known virtualization solution, is added on the top of the physical layer of the BS and is responsible for virtualizing the BS and the spectrum as well. In summary, the hypervisor accomplishes two tasks:

1) Hosts several virtual BSs onto a physical BS.

2) Schedules the wireless resources (PRBs) among the different virtual BSs.

Following this way, two different versions of the hypervisor exist [17]:

1) Static version: the hypervisor allocates the PRBs among the different tenants just once at the beginning. The number of the allocated PRBs for each tenant is equal, where each virtual BS will get the exact same 
amount of PRBs and keeps it regardless if it is being actually used or not.

2) Dynamic version: the PRBs are allocated to the different tenants in a dynamic manner at equal time intervals. The amount of the allocated PRBs will depend on the load that each tenant is experiencing during the last time instance. In this way, each operator will only get his required share of the PRBs and less waste of resources will occur.

Decades of experience with Internet has reinforced a general rule of thumb: it is nearly always more preferable to dynamically allocate resources over static resource allocations. Dynamic resource allocation can allow for more efficiency and flexibility in situations where the demand on scarce resources is not predictable. The multi-tenant model defines that each tenant is dynamically assigned and reassigned all the physical and virtual resources according to its consumers' demand [18].

This solution uses the PRB as the minimum resource granularity that can be allocated, and assigns PRBs among the different virtual nodes, and not among the users (as typically done by a scheduler). The PRBs are scheduled to the different virtual BSs based on previously arranged contracts (SLAs), which specify different guarantees for the operator owning a virtual BS. After the hypervisor allocates PRBs to the virtual BSs, each virtual BS allocates the PRBs to its users. In other words, the hypervisor is responsible for scheduling the air interface (between BSs and user equipment) resources (e.g., OFDMA sub-carriers).

Some other works, trying to avoid such a low-level strategy as in [13], propose mechanisms that schedule the resources between slices in a higher layer. This approach is generally done at the MAC layer or at the Network-layer. Such kind of work is the solution proposed in [15], where the authors define the Network Virtualization Substrate (NVS) that is a substrate on BSs enabling effective virtualization of the wireless resources. The NVS integrates virtualization into the WiMAX base station uplink/downlink scheduler software.

For efficient resource allocation, the BS includes a collection of schedulers. More specifically, the NVS is designed as a hierarchical scheduler divided in two steps, slice scheduling and flow scheduling:

- Slice scheduling is the process of selecting which slice has to transmit at every moment. Every slice can request a certain amount of resources or bandwidth. Given that by maximizing the total utility of the slices directly maximizes the revenue of the $\mathrm{MNO}$, the slice that maximizes the total utility will be selected.

- Flow scheduling can be customized by the selected slice (controlled by each tenant), selecting this way which flow should transmit in the first position. Basically, a traffic classifier splits packets in flows, according to their different QoS. More specifically, a downlink flow scheduler determines the sequence of packets to be transmitted in the downlink direction based on flow priorities, like VoIP traffic, video traffic, file sharing, and Web traffic with decreasing priority.
Similarly, an uplink flow scheduler determines uplink slot allocation based on the bandwidth requests from clients, channel quality, and QoS.

Finally, when both decisions have been taken, the frame scheduler will be invoked to perform the allocation of the resources to the resulting packet (mapping PRBs to specific slots in the MAC frame).

A similar framework is demonstrated in [19]. However, those researchers claim that instead of performing prioritization between different applications for each SP separately after allocation of RBs (postallocation priority), prioritization between different traffic types should be performed for the multiple Service Providers (SPs) jointly prior to the allocation of resources (pre-allocation priority). The reason is that a SP would possibly have insufficient resources to serve all real-time (RT) requests if the scheduler assigns resources on a fair-throughput basis without considering the traffic heterogeneity.

In another work [16], the authors take one step further and propose a spectrum-sharing framework to exploit virtualization in real LTE networks. They introduce the Virtual Resource Manager (VRM), which is responsible for collaborative spectrum allocation for different tenants, as a replacer of the LTE Radio Resource Manager (RRM) in a virtual network, in order to improve the system performance in a multi-tenant collaborative network. The VRM includes the fundamental radio resource scheduling feature, which distributes radio resources among Mobile Units (MUs), taking into account channel conditions and QoS requirements. The researchers develop an innovative approach called Time Domain Muting (TDM) Radio Resource Virtualization (RRV). This approach combines Radio Resource Virtualization (RRV) and enhanced Inter-Cell Interference Coordination (eICIC) to improve spectrum usage while protecting SPs from excessive interference. RRV, eICIC, and HetNet as well, are shortly explained below.

A HetNet consists of a mixture of different base station types such as high power base stations (macro cell) and low power base stations (small cell). Small cell BSs are typically deployed in hotspots to offload traffic from the macro cell. So, HetNet is regarded as an efficient solution to increase the network capacity in order to be able to carry the forecasted increase of data traffic [20].

For explaining the different spectrum allocation schemes, the following example is used [21]:

A simple two-cell model is used in a virtualized HetNet. It consists of the BS-A of a large cell, belonging to SP-A, and a small cell of SP-B that is located within BS-A coverage. A traditional network would distribute distinct spectrum bands for each SP in these two cells. In contrast, a virtualized network does not separate the bandwidth.

In the case of Separate Spectrum Virtualization (SSV), SP-A and SP-B can get varying amounts of spectrum every time interval based on their requirements, agreements, and policies. In SSV, the spectrum slices allocated to the two SPs in the same time interval do not overlap. This is an easy way to ensure 
isolation, but it does not make full use of the complex characteristic of spectrum since it creates an interference-free set of spectrum slices.

In the case of Radio Resource Virtualization (RRV), considering the radio resources as a function of geography and signal strength, it seems that the spectrum can be reused with varying levels of interference. This means that the same slice of spectrum can be reused by multiple SPs in the same time interval in overlapping geographical areas (that is, there is some interference).

One of the technical challenges, that needs to be addressed in order to fully benefit from HetNet deployments, is the interference management. Thus, the technique proposed is called eICIC with TDM muting for downlink co-channel deployment of macro and small cells [20].

The basic principle of TDM eICIC is to prevent the macro cell BSs from transmitting on certain subframes, meaning that they are periodically muted. During these subframes, no data signal will be sent from the macro cell BSs. As a result, the small cell BSs can schedule MUs, which would otherwise experience too high interference from the macro cell BS. However, the macro-BS should still transmit critical system information and Common Reference Signals (CRS).

\section{SDN and Multi-tenancy}

In the previous section, the state-of-the-art in mobile networks virtualization was provided. Below, the same concepts are extended to IP networks for enabling multitenancy.

SDN can be deployed on any traditional network environment, from home and enterprise networks to data centers. As the recent research on SDN shows that it is a promising technology, different commercial virtualization platforms based on SDN concepts have started to appear.

VMWare has proposed a network virtualization platform (NVP) [22] that provides the necessary abstractions to allow the creation of independent virtual networks for large-scale multi-tenant environments. NVP is a complete network virtualization solution that allows the creation of virtual networks, each with independent service model, topologies, and addressing architectures over the same physical network. With NVP, tenants do not need to know anything about the underlying network topology, configuration, or other specific aspects of the forwarding devices. NVP's network hypervisor translates the tenants' configurations and requirements into low-level instruction sets to be installed on the forwarding devices.

IBM has also recently proposed SDN VE [23], another commercial and enterprise-class network virtualization platform. SDN VE uses OpenDaylight as one of the building blocks of the so-called softwaredefined environments (SDEs). This solution also offers a complete implementation framework for network virtualization. Like NVP, it uses a host-based overlay approach, achieving advanced network abstraction that enables application-level network services in large-scale multitenant environments.

FlowVisor [24] is one of the early technologies to virtualize an SDN. Its basic idea is to allow multiple logical networks share the same OpenFlow networking infrastructure. For this purpose, it provides an abstraction layer that makes it easier to slice a data plane based on off-the-shelf OpenFlow-enabled switches, allowing multiple and diverse networks to coexist. Five slicing dimensions are considered in FlowVisor: bandwidth, topology, traffic, device CPU, and forwarding tables. Moreover, each network slice supports a controller, i.e., multiple controllers can coexist on top of the same physical network infrastructure. Each controller is allowed to act only on its own network slice. In general, a slice is defined as a particular set of flows on the data plane. From a system design perspective, FlowVisor is a transparent proxy that intercepts OpenFlow messages between switches and controllers. It partitions the link bandwidth and flow tables of each switch. Each slice receives a minimum data rate, and each guest controller gets its own virtual flow table in the switches.

Similar to FlowVisor, OpenVirteX [25] acts as a proxy between the NOS and the forwarding devices. However, its main goal is to provide virtual SDNs through topology, address, and control function virtualization. All these properties are necessary in multitenant environments where virtual networks need to be managed and migrated according to the computing and storage virtual resources. Virtual network topologies have to be mapped onto the underlying forwarding devices, with virtual addresses allowing tenants to completely manage their address space without depending on the underlying network elements addressing schemes.

AutoSlice [26] is another SDN-based virtualization proposal. Differently from FlowVisor, it focuses on the automation of the deployment and operation of virtual SDN (vSDN) topologies with minimal mediation or arbitration by the substrate network operator. Additionally, AutoSlice targets also scalability aspects of network hypervisors by optimizing resource utilization and by mitigating the flow-table limitations through a precise monitoring of the flow traffic statistics. Similarly to AutoSlice, AutoVFlow [27] also enables multidomain network virtualization. However, instead of having a single third party to control the mapping of vSDN topologies, as is the case of AutoSlice, AutoVFlow uses a multiproxy architecture that allows network owners to implement flow space virtualization in an autonomous way by exchanging information among the different domains.

FlowN [28] is based on a slightly different concept. Whereas FlowVisor can be compared to a full virtualization technology, FlowN is analogous to a container-based virtualization, i.e., a lightweight virtualization approach. FlowN was also primarily conceived to address multitenancy in the context of cloud platforms. It is designed to be scalable and allows a unique shared controller platform to be used for managing multiple domains in a cloud environment. 
Each tenant has full control over its virtual networks and is free to deploy any network abstraction and application on top of the controller platform.

\section{Conclusion}

Cellular technology is expected to be a critical tool for future connectivity. In $5 \mathrm{G}$ cellular networks of the future, virtualization and SDN are expected to be on the frontline, and it is a challenge to find ways to exploit them to handle the vast increase in data traveling across both the access and the core network. The IoT era will require extended automation of network functions, via virtualization, QoS-aware differentiation of different classes of IoT traffic, and the collection and analysis of data to enable virtualization and SDN to optimize the network. It is thus imperative for the future $5 \mathrm{G}$ architectural models to be designed having in mind the IoT data explosion.

After reviewing the proposed approaches above, some conclusions can be done for the design of next wireless virtualization solutions. As it has been shown throughout the state-of-the-art review, virtualization can be done at different parts of the network and also different levels: flow level, sub-carrier or time slot level, or even at the lowest level of hardware components. Virtualization at higher levels leads to a better multiplexing of resources across slices (and hence increased utilization with fluctuating traffic), and simplicity of implementation, but at the same time can reduce the efficiency of isolation and the flexibility of resource customization. Whereas, virtualization at lower level leads to a reverse effect.

As described throughout this work, there have been recent efforts to introduce wireless network virtualization, explain its performance requirements, architecture, uses cases and potential approaches to challenges. While both industry and academia embrace virtualization at unprecedented speeds, the development is still at an early stage, with many open questions. Although the combination of SDN with virtualization in future wireless access networks is expected to support the anticipated vast increase in the number of mobile devices, the heterogeneity in devices, requirements, and usage scenarios, leaves many hurdles yet to be taken. There are important unexplored research challenges such as resource management, inter-operability, instantiation, heterogeneity support, which should be addressed in order to realize an a virtualized $5 \mathrm{G}$ network that facilitates efficient resource allocation and multitenancy.

This work has received funding from the European Union Horizon 2020-MCSA-ITN-2015 Innovative Training Networks (ITN) under grant agreement No 675806 (5G-AURA) and No 641985 (5G-Wireless).

\section{References}

1. CISCO White Paper, Cisco visual networking index: Global mobile data traffic forecast update, 20162021, (2017)

2. A.J. Staring, Applying the Cloud Computing Model in LTE based Cellular Systems, (2012)

3. M. Richart, et al., Resource Slicing in Virtual Wireless Networks: A Survey, IEEE Trans. on Network and Service Management, 13, 15 (2016)

4. N. Bizanis, F.A. Kuipers, SDN and Virtualization Solutions for the Internet of Things: A Survey, IEEE Access, 4, 16 (2016)

5. E. Hossain, M. Hasan, $5 G$ Cellular: Key enabling technologies and research challenges, IEEE Instrumentation \& Measurement Mag, 18, 11 (2015)

6. S. Sun, et al., Integrating network function virtualization with SDR and SDN for $4 G / 5 G$ networks, IEEE Network, 29, 6 (2015)

7. Y. Li, M. Chen, Software-Defined Network Function Virtualization: A Survey, IEEE Access, 3, 12 (2015)

8. P. Rost, et al., Benefits and Challenges of Virtualization in $5 G$ Radio Access Networks, IEEE Communications Mag., 53, 8 (2015)

9. J. Cadir, et al., "Resource Pooling for Wireless Networks: Solutions for the Developing World", ACM SIGCOMM Computer Communication Review, 46, 6 (2016)

10. P. Rost, et al., "Benefits and Challenges of Virtualization in 5G Radio Access Networks," IEEE Comms Magazine, 53, 8 (2015)

11. M. Richart, et al., Resource Slicing in Virtual Wireless Networks: A Survey, IEEE Trans. on Network and Service Management, 13, 15 (2016)

12. S.S. Hong, et al., Picasso: Flexible $R F$ and Spectrum Slicing, Proc. of the ACM SIGCOMM, 12 (2012)

13. Y. Zaki, et al., LTE Wireless Virtualization and Spectrum Management, WMNC, (2011)

14. C. Liang, F.R. Yu, Wireless Virtualization for next generation mobile cellular networks, IEEE Wireless Comms, 22, 9 (2015)

15. R. Kokku, et al., NVS: A Substrate for Virtualizing Wireless Resources in Cellular Networks, IEEE/ACM Trans. on Networking, 20, 14 (2012)

16. X. Wang, et al., A Collaborative Spectrum-Sharing Framework for LTE Virtualization, IEEE Conf. on CIC, (2015)

17. Y. Zaki, et al., LTE mobile network virtualization: Exploiting multiplexing and multi-user diversity gain, Jour. Mobile Networks and Applications, 16, 9 (2011)

18. J. Cadir, et al., Resource Pooling for Wireless Networks: Solutions for the Developing World, ACM SIGCOMM Comp. Comm. Rev., 46, 6 (2016) 
19. A. Abdelhamid, et al., Resource Allocation for Heterogeneous Traffic in LTE Virtual Networks, IEEE Int. Conf. on MDM, (2015)

20. Y. Wang, K.I. Pedersen, Performance Analysis of Enhanced Inter-cell Interference Coordination in LTE-Advanced Heterogeneous Networks, IEEE $75^{\text {th }}$ VTC Spring, (2012)

21. X. Wang, et al., On Radio Resource Sharing in Multi-Antenna Virtualized Wireless Networks, Proc. of ACM Int. Workshop on MSWiM 2013, 10 (2013)

22. T. Koponen, et al., Network virtualization in multitenant datacenters, in Proc. 11th USENIX Symp. Netw. Syst. Design Implement., 14 (2014)

23. S. Racherla, et al., Implementing IBM Software Defined Network for Virtual Environments, Durham, NC, USA: IBM RedBooks, (2014)

24. R. Sherwood, et al., Can the production network be the testbed? , in Proc. 9th USENIX Conf. Oper. Syst. Design Implement., 6 (2010)

25. A. Al-Shabibi, et al., OpenVirteX: A Network Hypervisor, (2014)

26. Bozakov, P. Papadimitriou, AutoSlice: Automated and scalable slicing for software-defined networks, in Proc. ACM Conf. CoNEXT Student Workshop, 2 (2012)

27. H. Yamanaka, et al., AutoVFlow: Autonomous virtualization for wide-area OpenFlow networks, in Proc. 3rd Eur. Workshop Softw. Defined Netw., (2014)

28. D.A. Drutskoy, Software-defined network virtualization with FlowN, Ph.D. dissertation, Dept. Comput. Sci., Princeton Univ., Princeton, NJ, USA, (2012) 